\title{
Automaton model of protein: dynamics of conformational and functional states
}

\author{
Andrei Khrennikov and Ekaterina Yurova \\ International Center for Mathematical Modeling \\ in Physics and Cognitive Sciences \\ Linnaeus University, Växjö, S-35195, Sweden \\ Andrei.Khrennikov@lnu.se, Ekaterina.Yurova@lnu.se
}

July 10, 2018

\begin{abstract}
In this conceptual paper we propose to explore the analogy between ontic/epistemic description of quantum phenomena and interrelation between dynamics of conformational and functional states of proteins. Another new idea is to apply theory of automata to model the latter dynamics. In our model protein's behavior is modeled with the aid of two dynamical systems, ontic and epistemic, which describe evolution of conformational and functional states of proteins, respectively. The epistemic automaton is constructed from the ontic automaton on the basis of functional (observational) equivalence relation on the space of ontic states. This reminds a few approaches to emergent quantum mechanics in which a quantum (epistemic) state is treated as representing a class of prequantum (ontic) states. This approach does not match to the standard protein structure-function paradigm. However, it is perfect for modeling of behavior of intrinsically disordered proteins. Mathematically space of protein's ontic states (conformational states) is modeled with the aid of $p$-adic numbers or more general ultrametric spaces encoding the internal hierarchical structure of proteins. Connection with theory of $p$-adic dynamical systems is briefly discussed.
\end{abstract}


keywords: proteins, conformational and functional states, structure-function paradigm, automaton-model, quantum-like model

\section{Introduction}

In this note we apply automata theory [1, [2, [3] to modeling dynamics of proteins' states. The protein automaton model is presented by exploring the scientific methodology which plays the important role in foundations of quantum physics. This methodology is based on joint exploring of the two descriptive levels: ontic and epistemic. The ontic description is about reality "as it is" and the epistemic description is about observations, see Atmanspacher et al. [4]-[6], see also [7]- 9]. Now we want to apply this methodology to molecular biology. Conformation states of proteins are treated as ontic states and functional states or states approachable for observations as epistemic states. Such a separation of two descriptive levels is very fruitful in creation of an adequate model of dynamics of proteins' states. Moreover, it matches well with automata theory [1]: automata without output represent dynamics of ontic states, in our case conformational states, and automata with outputs represent dynamics of epistemic states, in our case functional/observational states.

We do not pretend that proteins are genuine quantum systems; we just want to use the methodology of ontic-epistemic states designed for quantum mechanics, see appendix 1 for discussion. The starting point linking dynamics of proteins with factorization models of quantum phenomena is the observation that the space of conformational states of a protein is huge and the concrete conformational states can be unobservables. Thus, as in quantum physics, one has to proceed with two types of states: ontic states (conformational states of a protein) and epistemic states (observable states representing various functions of a protein). We remark that, for a protein, the number of epistemic states is small, since each protein is a highly specialized biological machine with a few functions. At the same time the number of conformational states is huge.

In short we plan to model protein's state dynamics as functioning of an automaton without output having a huge configuration space (of conformational states); by adding output to this automaton we construct the model of observations of protein's functional states (epistemic states). Such an obser-

vational output produces factorization of the space of conformational states 
and can be mathematically represented as new "factorized automaton". In the framework of this automaton-model we discuss a number of conceptual problems related to production of functional states of proteins from conformational states. We emphasize that this is a concept-type paper. Our main aim is to present the concept of the automaton-protein. We also rise a series of questions which have to be clarified to make this model closer to the real biological situation, section 7. We plan to work on these questions in coming publications.

This model of protein's behavior does not match the standard protein structure-function paradigm formulated more than one hundred years ago [10], [11]. (In the operational terms by this paradigm the ontic and epistemic descriptions can be unified in the straightforward manner.) At the same time the ontic/epistemic operational approach is perfect for modeling of behavior of intrinsically disordered proteins [12], see section 2 for the discussion on recent studies of the molecular biological features of proteins' functioning.

We want that the paper will be readable for biologists. Therefore we try to minimize the mathematical apparatus in the use. The basic mathematical formalism under consideration is theory of automata, see [1] and section 3 for definition. We illustrate our model by a few illustrative examples, see Figures 1-3.

The presented model can be formulated in more advanced mathematical terms; in particular, by formalizing the mathematical structure of the ontic state space. We discuss shortly this problem appendix 2. The most natural model of proteins' state space is based on so called $p$-adic numbers, where $p>1$ is a natural number. For $p$ prime, these numbers are widely used in theory of dynamical systems, physics, biology and psychology [13][36]. We remark a kind of similarity between the $p$-adic state representation of protein's state dynamics and application of $p$-adic dynamical systems to encryption of data, see Anashin [13, 16, 18]. Thus one may speculate that nature encrypted functioning of proteins by using methods of $p$-adic cryptography. This is an interesting idea, but in this paper we cannot elaborate it in more detail (in particular, such elaboration would lead to exploration of advanced mathematics of theory of $p$-adic dynamical systems).

Finally, we remark that statistics of quantum observations has some peculiarities [37], see also appendix 1 with discussion on the Bell inequality. In physics it is known that automata-dynamics can generate some features of quantum statistics [4]. In this note we show that biological systems such as proteins (and others having huge configuration space of internal states and 
just a few functional/observational states) can demonstrate special statistical features (even generation of $p$-adic probabilities corresponding to the absence of statistical stabilization of frequencies of outputs, see [38], [15] for such probabilities and their role in non-Archimedean physics and biology).

\section{Dynamics of proteins conformational and functional states from the perspective of molecular biology}

In this section we apply the ontic-epistemic approach to state dynamics (which we borrow from quantum foundations) to the protein dynamics.

By the the protein structure-function paradigm, any protein function is determined by a fixed three-dimensional structure [11]. This paradigm has been dominating in molecular biology during more than one hundred years, see Fischer [10] for one of the pioneer studies. At the same time it was challenged during many years including experimental evidences of its violation. Nevertheless, it stayed firmly as the cornerstone of molecular biology; only the discovery of intrinsically disordered proteins (IDPs), Dunker et al. [12, Dyson and Wright [39, Dunker et al. [40] changed (at least partially) the common viewpoint on the structure-function isomorphism. Nowadays it is widely understood that "functioning proteins do not remain fixed in a unique structure, but instead they sample a range of conformations facilitated by motions within the protein", Ramanathan et al. [41], see also Henzler-Wildman et al. 42 .

We recall that the native state of a protein is its completely folded functional state. However, opposite to the protein structure-function paradigm, the native state is no uniquely determined by protein's geometry. We again cite Ramanathan et al. [41]:

"Even in the native state, a protein exists as a collection of interconverting conformations driven by thermodynamic fluctuations. Motions on the fast time scale allow a protein to sample conformations in the nearby area of its conformational landscape, while motions on slower time scales give it access to conformations in distal areas of the landscape."

It is important to point out that this is the general picture of protein's functioning and not only IDP's functioning.

This picture matches perfectly with the ontic-epistemic approach to the 
description of dynamics of physical and biological systems. The concrete conformational states of a protein are typically unapproachable by experimenters. From the modern viewpoint, the native state, the functional state, cannot be identified with the concrete conformational state. It represents an ensemble of conformational states. Moreover, the native state is a fuzzy notation for a family of functional substates and protein's functioning can be represented as the dynamics in the space of these substates. Each of them corresponds to a special subensemble of conformational states. "Emerging evidence indicates that protein landscapes contain conformational substates with dynamic and structural features that support the designated function of the protein", 41]. In this paper we shall identify such "substates" with the functional states of a protein.

Thus we have the two levels description of protein's dynamics:

- conformational states dynamics: fast and stochastic;

- functional states dynamics: slower and deterministic

(see, e.g., Henzler-Wildman et al. [42] on time scales in protein dynamics).

One of the main consequences of the experimental and theoretical studies is that the dynamics of conformational states, in spite of its stochastic nature, has the functional meta-meaning, i.e., it is not reduced to thermodynamical fluctuations. This is a delicate point and we shall try to explain it in more detail. Physically there are, of course, thermodynamical fluctuations. But proteins were able to shape these fluctuations in the functional way - to perform transitions (through long chains of rapid conformations) from one functional state to another, see Hammes et al. [43], Ramanathan and Agarwal [44], Ramanathan et al. [45].

Nuclear magnetic resonance devices can be used to determine ensembles of conformational states of proteins. This is not the end of the experimental studies: X-ray crystallography allows researchers to identify the most populated states along the landscape. In the functional cycles of proteins, ensembles of conformational states (determining functional sates) are transferred from one to another. Such ensemble transformations are approachable for the experimental inspection. However, the dynamics of conformational states in subensembles determining functional states and their jumps from one ensemble to another cannot be detected, at least by existing measurement devices, cf. again with the situation in quantum physics. 
We emphasize that in contrast to quantum physics, in molecular biology there are no doubts in the existence of conformational states in a protein "as it is" (although in general they are not accessible experimentally). Of course, there is the crucial difference between molecular biology and quantum physics. In biology it is possible "to see" the spatial structure of a protein and concrete geometric figures can be used for labeling of conformational states. In quantum physics, the existing measurement devices do not provide the possibility of determination of a similar fine structure.

\section{Automaton model of protein}

As a model of the protein dynamics, we consider an automaton [1] $\mathfrak{A}=$ $\langle I, S, V, f, g\rangle$, where $I(V)$ is input (output) alphabet, $S$ is the set of states, $f: I \times S \rightarrow S$ and $g: I \times S \rightarrow V$ are function of transition and output, respectively, i.e., depending on the iteration of functioning of the automaton:

$$
\begin{gathered}
s_{k+1}=f\left(i_{k}, s_{k}\right), k \geq 0 . \\
v_{k}=g\left(i_{k}, s_{k}\right), k \geq 0 .
\end{gathered}
$$

At the moment $n=0$ the automaton $\mathfrak{A}$ is at the state $s_{0}$; once fed by the input symbol $i_{0} \in I, \mathfrak{A}$ outputs the symbol $v_{0}=g\left(i_{0}, s_{0}\right) \in O$ and reaches the state $s_{1}=f\left(i_{0}, s_{0}\right) \in S$; then $\mathfrak{A}$ is fed by the next input symbol $i_{1} \in I$ and repeats the routine.

The automaton $\mathfrak{A}$ transfer words with respect to the alphabet $I$ to words with respect to the alphabet $V$. This map, defined on the set of $I$-words and taking values in the set of $V$-words and determined with the aid of two functions, $f$ and $g$, i.e., associated with the automaton $\mathfrak{A}$, is denoted as $\mathfrak{F}_{\mathfrak{A}}$.

To construct the automaton model of the protein dynamics, we have to describe its basic parameters:

- States. We assume that a protein consists of $N$ connected elementary units, e.g., molecules, and that each of them has $p$ degrees of freedom. Here $p>1$ is a natural number. Each conformational state of a protein is represented as the vector

$$
\vec{s}=\left(x_{0}, \ldots, x_{N-1}\right), x_{i} \in F_{p}=\{0,1, \ldots, p-1\} .
$$


- Input and output alphabets. The input alphabet represents the impact of protein's environment, e.g., temperature, ferments, chemical molecules around it. In our mathematical model it is assumed that influences of the environment can be represented numerically, as the elements of the set $I$. The output alphabet represents the results of observations on a protein; these results are represented as elements of the set $V$.

- Functions. The function $f$ is determined by the structure of a concrete protein. The function $g$ is selected depending on the way of observation on a protein: the input alphabet (impact of the environment), the space of states $S$, and the transition function $f$.

Thus in our model protein by itself is represented as an automaton without output. The output represents observations. Their aim is to reconstruct protein's parameters.

We remark that automata with finite state space $S=\left\{s_{1}, \ldots, s_{m}\right\}$ (and the fixed initial state $s_{0} \in S$ ) are known as the Mealy machines [46]. It is not clear whether a protein has to be modeled as a Mealy machine. Conformational states of protein are determined by a variety of physical parameters, including the geometric configurations. The space of such parameters can be infinite and even continuous. Of course, mathematically it is easier to proceed with Mealy machines.

\section{Factor-automaton from observed output se- quence}

We remark that the space $S$ of possible conformational states of a protein is huge, it contains $N_{\text {conf }}=p^{N}(N \sim 300)$ elements. Therefore to construct an automaton working on such a space and completely describing functioning of the concrete protein is practically impossible. Thus the existence of such a complete automaton has basically only a theoretical value. Possible observations cannot determine uniquely protein's state, one fixed among $N_{\text {conf }}$ states of $S$, at the instant of measurement, i.e., observations are not able to distinguish states from $S$.

In reality from observable data we can construct only some factor-automaton for the "complete automaton" $\mathfrak{A}$ (ontic automaton), 1 To construct the factor-

\footnotetext{
${ }^{1}$ This situation reminds emergence of the quantum model, treated as an operational
} 
automaton representing observations, we consider the following equivalence relation $\rho$ on the set $S$. The states $s_{1}, s_{2}$ are $\rho$-equivalent, non-distinguishable with respect to observations, if

$$
g\left(i, s_{1}\right)=g\left(i, s_{2}\right) \forall i \in I .
$$

The factor-set $S_{\rho}$ of $S$ with respect to $\rho$ is considered as the space of states of the new automaton $\mathfrak{A}_{\rho}$. The functions $f$ and $g$ have to "respect" the equivalence relation $\rho$, i.e., if

$$
s_{1} \rho s_{2}
$$

then

$$
f_{\rho}\left(i, s_{1}\right)=f_{\rho}\left(i, s_{2}\right), g_{\rho}\left(i, s_{1}\right)=g_{\rho}\left(i, s_{2}\right), i \in I .
$$

The $\mathfrak{A}_{\rho}=\left\langle I, S_{\rho}, V, f_{\rho}, g_{\rho}\right\rangle$ is called the factor-automaton of the original automaton $\mathfrak{A}$.

Thus at the level of observation conformational states of a protein have to be unified in some classes, cf. again with ontic-epistemic approach to quantum theory.

The same model can be used in more concrete biological framework (concertizing the above general observational model). We can consider a protein as well specialized biological machine which performs a few concrete functions. In terms of automaton's states this viewpoint means that on the space of conformational states $S$ it is possible to define some equivalence relation which is determined by protein functions. Such a functionality-factorization also leads to the factor-automaton of the original protein-automaton. Thus the states of the factor-automaton can be treated as functional states of a protein.

Fig. 1 illustrates construction of factor-automata. At this figure the ovals $C_{1}, C_{2}, C_{3}, C_{4}, C_{5}$ represent classes of equivalence with respect to an equivalence relation defined on the space of all possible states $S$; in section 5 we shall present detailed construction of the concrete factor-automaton.

observation model, from prequantum models; generation of epistemic state description from the ontic state description, see the introduction. 


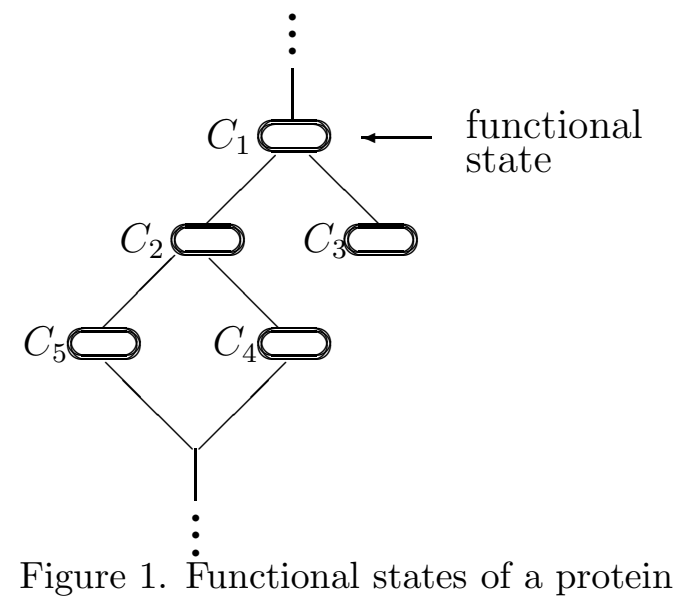

In future the set of states of the automaton $\mathfrak{A}$ belonging to some equivalence class with respect to aforementioned equivalence relation we shall call a generalized state of the automaton. This terminology is useful to have the possibility to consider states of the factor-atomaton $\mathfrak{A}_{\rho}$ as sets. In another terminology generalized states are basins, i.e., a basin can be considered as a class of equivalence with respect to some equivalence relation $\rho$.

\section{Example of construction of factor automa- ton}

Let us consider automaton $\mathcal{A}=\langle I, S, Y, f, F\rangle$, where $I=\{0,1,2\}$ denotes the input alphabet, $Y=\{a, b, c, d\}$ denotes the output alphabet, and $S=$ $\left\{s_{1}, s_{2}, s_{3}, s_{4}, s_{5}, s_{6}, s_{7}, s_{8}\right\}$ is the state space. The transition function $f$ and the output function $g$ are given by Table 1 .

Table 1. Transition and output functions for the original automaton.

Table 1 shows that, for this automaton, output depends only on the state. Such automata are known as the Moore machines, see section 9.1, The diagram of transitions of the (Moore) automaton determined by Table 1 is represented on the graph of Fig. 2. We repeat that here $g: S \rightarrow V$, i.e., it is independent of the input symbol $i$. 


\begin{tabular}{|c|c|c|c|c|c|c|c|c|}
\hline$f(i, s)$ & \multicolumn{10}{|c|}{$(\mathrm{s})$} \\
\hline$(\mathrm{i})$ & $s_{1}$ & $s_{2}$ & $s_{3}$ & $s_{4}$ & $s_{5}$ & $s_{6}$ & $s_{7}$ & $s_{8}$ \\
\hline 0 & $s_{1}$ & $s_{3}$ & $s_{1}$ & $s_{4}$ & $s_{4}$ & $s_{7}$ & $s_{8}$ & $s_{6}$ \\
\hline 1 & $s_{3}$ & $s_{1}$ & $s_{2}$ & $s_{4}$ & $s_{2}$ & $s_{5}$ & $s_{5}$ & $s_{5}$ \\
\hline 2 & $s_{4}$ & $s_{4}$ & $s_{4}$ & $s_{4}$ & $s_{7}$ & $s_{8}$ & $s_{6}$ & $s_{8}$ \\
\hline$g(i, s)$ & \multicolumn{10}{|c|}{$(\mathrm{s})$} \\
\hline$(\mathrm{i})$ & $s_{1}$ & $s_{2}$ & $s_{3}$ & $s_{4}$ & $s_{5}$ & $s_{6}$ & $s_{7}$ & $s_{8}$ \\
\hline 0 & $a$ & $a$ & $a$ & $b$ & $c$ & $d$ & $d$ & $d$ \\
\hline 1 & $a$ & $a$ & $a$ & $b$ & $c$ & $d$ & $d$ & $d$ \\
\hline 2 & $a$ & $a$ & $a$ & $b$ & $c$ & $d$ & $d$ & $d$ \\
\hline
\end{tabular}

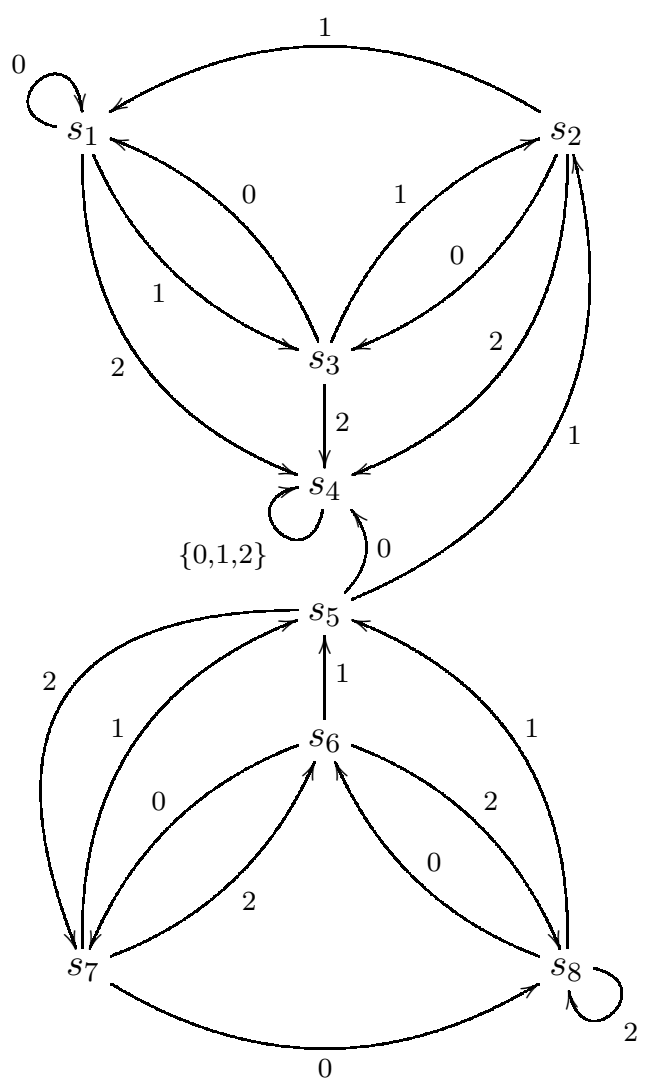

Figure 2. Transition diagram of the original automaton.

We consider the following equivalence relation $\rho$ on the set of states. It is determined with the aid of the disjoint partition of the set of states: $S=$ $\left\{s_{1}, s_{2}, s_{3}\right\} \cup\left\{s_{4}\right\} \cup\left\{s_{5}\right\} \cup\left\{s_{6}, s_{7}, s_{8}\right\}$. 
The equivalence relation $\rho$ is consistent with the transition function $f$, see Table 1, i.e. if $s_{\alpha} \rho s_{\beta}$, then $f\left(i, s_{\alpha}\right) \rho f\left(i, s_{\beta}\right), i \in I ; \rho$ is also consistent with the output function $g$.

Now consider the new automaton $\mathcal{A} / \rho=\left\langle I, S / \rho, Y, f_{\rho}, g_{\rho}\right\rangle$, factor-automaton with respect to $\rho$, which is consistent with the transition and output 'functions (congruence of the original automaton), where

$$
S / \rho=\left\{S_{1}=\left\{s_{1}, s_{2}, s_{3}\right\}, S_{2}=\left\{s_{4}\right\}, S_{3}=\left\{s_{5}\right\}, S_{4}=\left\{s_{6}, s_{7}, s_{8}\right\}\right\},
$$

and the transition function $f_{\rho}$ and the output function $g_{\rho}$ are given by Table 2 .

\begin{tabular}{|c|c|c|c|c|}
\hline$f_{\rho}$ & \multicolumn{5}{|c|}{$(S)$} \\
\hline$(\mathrm{i})$ & $S_{1}$ & $S_{2}$ & $S_{3}$ & $S_{4}$ \\
\hline 0 & $S_{1}$ & $S_{2}$ & $S_{2}$ & $S_{4}$ \\
\hline 1 & $S_{1}$ & $S_{2}$ & $S_{1}$ & $S_{3}$ \\
\hline 2 & $S_{4}$ & $S_{2}$ & $S_{4}$ & $S_{4}$ \\
\hline$g_{\rho}$ & \multicolumn{5}{|c|}{$S)$} \\
\hline$(\mathrm{i})$ & $S_{1}$ & $S_{2}$ & $S_{3}$ & $S_{4}$ \\
\hline 0 & $a$ & $b$ & $c$ & $d$ \\
\hline 1 & $a$ & $b$ & $c$ & $d$ \\
\hline 2 & $a$ & $b$ & $c$ & $d$ \\
\hline
\end{tabular}

Table 2. Transition and output functions for the

factor automaton.

The diagram of transitions of the automaton $\mathcal{A} / \rho$ determined by Table 2 is presented at Fig. 3.

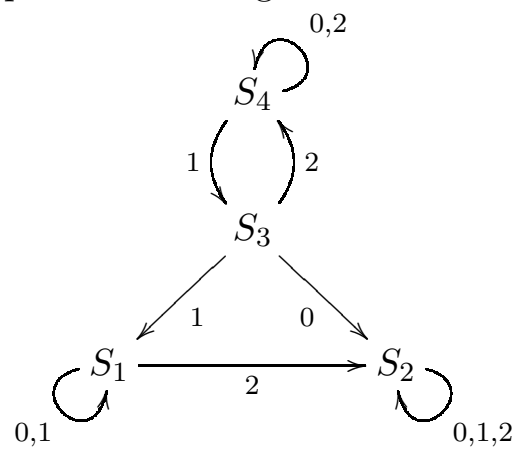

Figure 3. Transition diagram of the factor automaton.

\section{Protein as a Moore machine?}

As was remarked in the previous section, the automaton determined by Table 1 and Fig. 2 belongs to the class of automata known as the Moore machines 
[47]: a finite-state machine (which initial state is fixed) whose output values are determined solely by its current state. Such automata are widely used in computer science and digital electronics.

A bio-system modeled by a Moore machine used environmental inputs only to modify its internal states. Functional outputs are determined solely by the latter. Such machines, although perfectly adaptive to signals from the surrounding environment, have some degree of independence. First they handle environment's signal to modify the state and only later use this state to produce a functional output. Thus the information from outside is used not straightforwardly. We can say that it is "analyzed" inside the bio-system and only then used for the corresponding functional response. This is the reason to use the Moore machines to model protein's behavior.

We remark that many digital electronic systems work as Moore machines of the special type, so called clocked sequential systems. In such a system the state changes only when the global clock signal changes. This change of the state induces change of the output. Clocked sequential systems are one way to solve metastability problems.

We stress that a Moore machine is the finite state machine. However, as was already pointed out in section 3, we have reasons both in favor and against using the finite state automata for modeling protein's behavior. Therefore it is useful to consider so to say infinite state Moore machines. For such an automaton, its state space $S$ can be infinite and even continuous and the output function depend only on the state

The coupling of our protein model with computer science and digital electronics is the interesting topic for further speculations on the computational structure of functioning of cell's components and cells in general. Another important topic is comparison of our modeling of protein's functioning with mathematical modeling of intelligence, both biological and artificial. The basic model of artificial intelligence (or to be more rigorous computing) is given by the Turing machine. The latter is definitely more powerful than finite state automata, the Mealy machines and, in particular, the Moore machines. However, in our modeling of activity of a protein molecular we do not restrict consideration to the finite state automata. Comparison of behavioral features of bio-systems modeled with the aid of Turing machines and infinite automata is the interesting problem related to intelligence of bio-systems. In particular, it can be the starting point in development of theory of intelligence of proteins. 


\section{Problems related to analysis of observational data}

As was pointed out, the automaton model of a protein is an automaton without output. However, initially we do not know the structure of this automaton, i.e., the space of states, transition functions, and very often neither the input alphabet which encodes influence of environment on behavior of a protein. To reconstruct the parameters of the automaton model of a protein, we have to use the results of observations on protein's behaviour. For this aim, the automaton model of a protein is completed by the output alphabet $V$ and the output function $g$ which are determined by possibilities of observations.

Thus we consider an automaton with output $\mathfrak{A}=\langle I, S, V, f, g\rangle$, where $V$ is the output alphabet and $g I \times S \rightarrow V$ is the output function. As the result of functioning of this automaton with output we observe a sequence of the form:

$$
v_{k}=g\left(i_{k}, s_{k}\right), k \geq 0 .
$$

We remark that by itself the automaton model of a protein is considered as the automaton $\langle I, S, f\rangle$, where, as we shall consider in future, the set of states $S$ consists of generalized states.

From observation on the output sequence $v_{k}, k \geq 0$ of the automaton $\mathfrak{A}$ we can make conclusions about behavior of the protein-automaton as well as about its structure (states, transition functions, input sequences ans so on). In particular, we can consider the following problems:

- if the transition function $f_{\rho}$ is only partially defined, then from observations $v_{k}, k \geq 0$, it can be reconstructed;

- it is possible to reconstruct (determine) the input alphabet of an automaton, i.e., to check the influence of an input symbol on automaton's behavior;

- to check the presence of equivalent states by using the output sequence; in other words, to clarify the structure of the equivalence relation on the set of states, i.e., states of the form $\vec{s}=\left(s_{0}, \ldots, s_{N-1}\right)$;

- to check matching of an automaton with the output data (observed in experiments);

- to determine the initial state of the automaton $\mathfrak{A}$.

Since the automaton $\mathfrak{A}$ can have generalized states, its output sequences $v_{k}, k \geq$ 0 can have the following specific feature. An output sequence of the automaton 
$\mathfrak{A}$ can contain a long sequence of equal elements. Such sequences appear if the automaton is being in some fixed generalized state. If the output sequence is considered as realizations of some random variable $\gamma$, then the presence of long series can imply some "pathological features" of $\gamma$, e.g., $\gamma$ does not have the mathematical expectation. Such features make difficult construction of a proper statistical criteria for solution of aforementioned problems.

To illustrate this situation, we consider behavior of the automaton $\mathfrak{A}$ in a neighborhood of a generalized state $C$ as it is shown at Fig. 4.

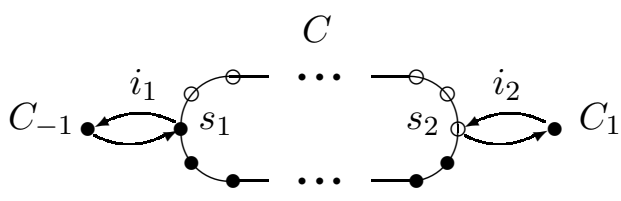

Figure 4. Unconventional probabilistic behavior.

There are three generalized states $C_{-1}, C, C_{1}$. Each of generalized states $C_{-1}, C_{1}$ contains just one conformational state. The generalized state $C$ consists of $2 p^{r}$, where $p>1$ is a natural number, conformational states (the states $s_{1}, \ldots, s_{2}$ belong to $C$ ). Conformation states composing $C$ form two special classes, at Fig. 4 they are represented by white and black spots. Each class contains $p^{r}$ conformational states.

The automaton is functioning in the following way. If it is in state $C_{-1}\left(C_{1}\right)$, then, for each input, the automaton is transferred into the conformational state $s_{1}$ $\left(s_{2}\right)$ belonging to the generalized state $C$. If the automaton is in one of conformational states composing $C$, excluding the states $s_{1}$ and $s_{2}$, and marked by white (black) points, then for each input the automaton is transferred into the closest from the left-hand (right-hand) side state. Thus in the generalized state $C$ the automaton goes through conformational states composing $C$ cyclically. For the states $s_{1}$ and $s_{2}$, its behavior is more complex. There are two possible transitions scenarios. If input symbol is $i_{1}\left(i_{2}\right)$, then the automaton jumps into the generalized state $C_{-1}\left(C_{1}\right)$. If the input symbol is different from $i_{1}\left(i_{2}\right)$, then the automaton stays in the generalized state $C$ and its state is changed in accordance with the general law of cyclic dynamics insied $C$.

Suppose now that the output function of the automaton $g$ depends only on the generalized state, i.e., it does not depend on input and automaton's output $v_{-1}$ is observed if the automaton is in the state $C_{-1}$ (respectively, $v$ and $v_{1}$ for $C, C_{1}$ ).

Since in the state $C$ the automaton can stay $\xi p^{r}$ steps of dynamics, where $\xi$ is some integer valued random variable, in general the relative frequency of appear- 
ance of the symbol $v$ in the output sequence can fluctuate, without stabilization to any constant - probability. Thus the probability $P(C)$ of the state $C$ is not well defined. Besides of this "probabilistic pathology" (violation of von Mises principle of stabilization of relative frequences), we point to another feature of behavior of relative frequencies: for the states $v_{-1}$ and $v_{1}$, they approach zero (when the length of the output sequence approaches infinity). Thus the probabilities of appearance of this automaton in the states $C_{-1}, C_{1}$ equal to zero. Therefore from the probabilistic viewpoint these states can be simply eliminated from the model. However, this will lead to a biologically inadequate model.

For such sequences of observations, we can use the apparatus of generalized probability theory based on representation of probabilities not by real numbers from the segment $[0,1]$, but by so called $p$-adic numbers, where $p>1$ is a prime number. In this framework the relative frequencies (which are always rational numbers) are embedded into the fields of $p$-adic numbers $\mathbf{Q}_{p}$ and their limits are considered in these fields. In this way the probabilities $P(C)$ and $P\left(C_{-1}\right), P\left(C_{1}\right)$ are well defined and the last two of them are nonzero. In this paper we cannot go in more details, see [38], [15].

\section{Automaton model: some features of pro- tein's behavior and interpretations}

In this section we shall consider some special features of protein's behavior implied by our automaton-model and possible interpretations of these features.

1. Testing the presence of generalized states. Consider the following situation represented at Fig. 5.1 and 5.2. In the first case the automanton has only two generalized states for which we observe outputs $v_{-1}$ and $v_{1}$, respectively. In the second case (Fig. 5.2) there is the generalized state $C$ between the states $C_{-1}$ and $C_{1}$. However, when this automaton is in the state $C$ no outputs are observed. If the automaton is in the state $C_{1}\left(C_{-1}\right)$, but has not yet moved to the state $C_{-1}\left(C_{1}\right)$, then the symbol $v_{1}\left(v_{-1}\right)$ is observed at the output sequence .

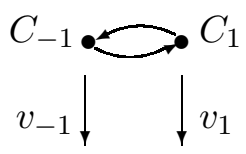

Figure 5.1. No intermediate state 


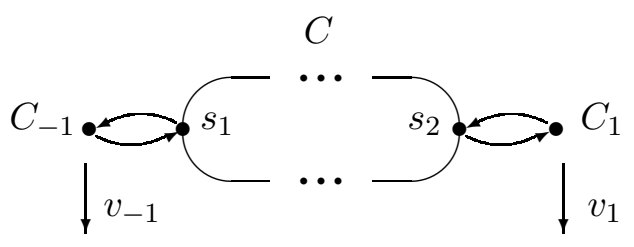

Figure 5.2. Intermediate state

The output sequence can be used to determine the presence of an intermediate state $C$ which can be present in a protein. The presence of such a state $C$ implies that the output sequence of the automaton presented at Fig. 5.2 contains series of elements $v_{-1}$ and $v_{1}$. The lengths of series can be considered as values of some random variable. In the case of the automaton presented at Fig. 5.1 the output sequence also consists of the elements $v_{-1}$ and $v_{1}$. In principle this sequence also can contain long series of these elements, but their probabilistic structure differs from the probabilistic structure of the series in the output sequence generated by the automaton of Fig. 5.2. We now point to one of the important marks of the presence of an intermediate state $C$ : in the sequence produced by the automaton of Fig. 5.2, the law of stabilization of relative frequencies of appearance of $v_{-1}$ and $v_{1}$ can be violated. As was remarked, for analysis of such statistical data, $p$-adic probability methods can be applied.

2. The presence of generalized states and protein's transition time. Our model with generalized states as composed of internal (conformational) states of proteins can explain the well known experimental fact that transition of a protein from one functional state to another can take long time and, moreover, that the interval of transition time can vary essentially from transition to transition, Henzler-Wildman et al. 42. Thus the descriptive level based on ontic states of proteins has some explanatory power for observable (epistemic) features of proteins.

3. Hierarchy. By using the automaton model of a protein the set of generalized states, functional states of proteins, can be endowed with hierarchic structures, in particular, tree-like. We remark that the feature of the model that transition from one functional state to another is performed through other generalized states (Ramanathan et al. [41]) can be explained even without appealing to a hierarchic structure in proteins. (For example, the graph represented at Fig. 6 is, in fact, the binary tree. However, the presence of the tree-like structure is not straightforwardly visible at Fig. 6.) To fill hierarchy with nontrivial content (i.e., not just the graphic scheme), perhaps we have 
to use some additional criteria. For example, a hierarchy determining rule can be based on the magnitude of the total energy of transition from one functional state to another or the magnitude of the potential energy barrier separating these states, cf. with works [].

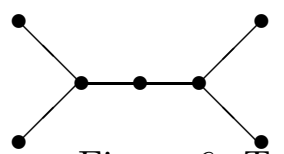

Figure 6. Tree-like structure of protein-automaton.

4. Functional inseparability. In the framework of the protein-automaton we can consider the following extremal case. For example, the factor-automaton consists of a single generalized state $C$ - the native state of a protein. In such a situation we are not able to separate functional abilities of conformational states. Even by knowing that the number of conformational states is huge we have the trivial factor-automaton. Moreover, the impossibility of epistemic splitting of $C$ strongly supports the protein structure-function paradigm. It is very attractive to extend this uniqueness from the epistemic level to the ontic level. And this happened at the first stage of development of molecular biology (in fact, the very long stage), see section 2 .

However, from the modern viewpoint [41], the space of conformational (ontic) states has to be divided into classes - generalized (epistemic) states. The latter states have to be distinguishable from the functional viewpoint (as representing observable behavior of a protein). The behavior of a protein inside a generalized state can be considered as "random and equiprobabilistic" and having no influence on functioning of a protein.

We remark that from the protein-automaton viewpoint the rather common thesis that proteins are biological machines posses the following constraint on the relative number of generalized states $N_{\text {gen }}$. It has to be small, but at the same time not too small. On one hand, if $N_{\text {gen }}$ is large, then such a biological machine has to perform a large number of various functions or if all these generalized states ("substates of the native state" in terminology of Ramanathan et al. [41]) are involved in a single functional cycle, then its performance will take too much time. This contradict to the experiment and the thesis that a protein is similar to a machine. On the other hand, if $N_{\text {gen }}$ is very small (e.g., just one as in the above example), then behavior of such a biological system is trivial (from the epistemological viewpoint). 


\section{Mathematical formalism}

In the previous sections we tried to minimize the use of the mathematical apparatus; in fact, we proceed with only one mathematical structure, automaton, representing states' dynamics. Now we want to be more precise in mathematical terms.

\section{1 $\quad P$-adic model of the space of conformational states}

For a mathematical model of the state space of the protein-automaton, we can consider infinite sequences (cf. with (3) ):

$$
\vec{s}=\left(x_{0}, \ldots, x_{N-1}, \ldots\right), x_{i} \in F_{p}=\{0,1, \ldots, p-1\} .
$$

Here $p>1$ is a natural number. Denote the space of such sequences by the symbol $\mathbf{Z}_{p}$. For mathematical modeling, it is useful to endow $\mathbf{Z}_{p}$. with an algebraic structure similar to the algebraic structure on real numbers. Each sequence is represented as the series of the form:

$$
\vec{s}=\sum_{i=0}^{\infty} x_{i} p^{i}, x_{i} \in F_{p} .
$$

We remark that the set of natural numbers $\mathbf{N}$ is the subset of $\mathbf{Z}_{p}$ consisting of finite sums (expansions of natural numbers with respect to the base $p$ ). Moreover, negative integers can be also represented in the form of such power series, by using so called complement code representation which is standard for computer science. For example, if $p=2$, then $-1=11111 \ldots . \ldots . .=\sum_{i=0}^{\infty} 2^{i}$. Thus even the set of integers $\mathbf{Z}$ can be embedded in $\mathbf{Z}_{p}$. The operations of addition, subtraction and multiplication can be extended from $\mathbf{Z}$ onto $\mathbf{Z}_{p}$. We obtain the algebraic structure called a ring. Division is in general not well defined, see, e.g., [15] for details.

The space $S=\mathbf{Z}_{p}$ can serve as the mathematical model of the (ontic) state space of the protein-automaton. It can be endowed with a metric which is so called ultrametric, see section 9.2. Thus the (ontic) state space of the protein-automaton is the ultrametric space. Ultrametricity expresses mathematically the hierarchic structure of the state space, see section 9.2. This is the simplest ultrametric mathematical model.

In a more general model the ranges of digits $x_{i}$ need not coincide, i.e., $x_{i} \in$ $F_{p_{i}}=\left\{0,1, \ldots, p_{i}-1\right\}$, where $p_{i}>1$ is a natural number. The latter model is more natural, since encoding unit $i$ in the digital representation of protein's state, see (3) and (5), can have its own degree of complexity reflected in the number $p_{i}$. We set $\mathbf{p}=\left(p_{0}, \ldots, p_{k}, \ldots\right)$. Denote the space of sequences with $x_{i} \in F_{p_{i}}$ by the symbol $\mathbf{Z}_{\mathbf{p}}$. 
This space can also be endowed with the algebraic structure and the ultrametric. Unfortunately, analysis on $\mathbf{Z}_{\mathbf{p}}$ is very complicated. In particular, nothing was done to develop theory of dynamical systems for such spaces. And the latter can serve as a tool to construct concrete examples of protein's state dynamics, see section 9.2 .

\subsection{Ultrametricity}

Typically a hierarchic structure in a physical or biological system can be mathematically presented as ultrametricity, i.e., the ultrametric space structure on the state space. The ultrametric model is widely used in theory of complex disordered systems (e.g., spin glasses) [22]-24], in genetics [25]- 27], cognition and psychology [28]-36]. We recall briefly the definition of an ultrametric space and the main features of its system of neighborhoods given by ultrametric balls. We start with the terminological remark: ultrametric spaces are also known as non-Archimedean spaces [15].

Let $S$ be a set (in our case the state space of a protein, the space of its conformational states). Let $d: S \times S \rightarrow \mathbf{R}_{+}$be a metric (distance). It is called an ultrametric if, besides the standard triangle inequality, it satisfies the strong triangle inequality:

$$
d(x, y) \leq \max [d(x, z), d(z, y)], x, y, z \in S .
$$

This inequality for a metric implies many unusual features of the system of neighborhoods in $S$ given by the balls

$$
U_{r}(a)=\{x \in S: d(x, y) \leq r\}, U_{r}^{-}(a)=\{x \in S: d(x, y)<r\},
$$

where $r>0$ and $a \in S$.

1. All balls are topologically open and closed at the same time - "clopen".

2. They are either disjoint or one is subset of another.

3. Any point $b$ belonging to the ball can be chosen as its center. Thus if $b \in U_{r}(a)$, then $U_{r}(a)=U_{r}(b)$. The same holds for balls $U_{r}^{-}(a)$. We can say that "all points of a ball-neighborhood have equal rights."

We now turn to our factor-automaton model of protein functioning. If we take any conformational state $s$ belonging to a functional state $C$, then $s$ completely determines the latter - as a representative of the factor class $C$. Consider the functional state $C$ as a neighborhood of the conformational state $s$. Then any $s \in C$ can be treated as the center of this neighborhood. Thus all conformational states 
belonging to a functional state "have equal rights" (from the observational viewpoint they produce the same output). This is an exhibition of ultrametricity of the state space of the automaton-protein. This is so to say epistemic ultrametricity, it is generated by functional factorization of conformational states. By using ultrametric spaces we can represent functional states by balls $C=U_{r}(s)$.

Consider the mathematical model in which the space of conformational states $S=\mathbf{Z}_{p}$ (the ring of $p$-adic integers) and all functional states are given by balls of the same radius $r=1 / p^{k}$. The set of all conformational states $S$ can be represented as the disjoint union of the balls of the radius $r: S=\cup_{j=0}^{p^{k}-1} U_{r}(j)$. This state space of balls can be obtained as the output of the factorization procedure on $S$ based on the $\bmod \mathrm{p}^{\mathrm{n}}$ equivalence relation: for $x, y \in \mathbf{Z}_{p}, x \sim y$ if and only if $x-y$ is divisible by $p^{n}$ (see (6) for number-theoretic presentation of elements of $\mathbf{Z}_{p}$ ).

Functioning of the protein-automaton can be described as a $p$-adic dynamical system [13]-18], see appendix 2.

\section{Conclusion}

We proposed to model protein behavior by using theory of automata.

We pointed out to similarity between modeling of behavior of proteins and quantum systems. In analysis of quantum foundations some authors explore the ontic-epistemic methodology, see Appendix 1. Quantum mechanics is treated as the epistemic (observational) model which can be emergent from a causal ontic (subquantum) model, see, e.g., Atmanspacher et al. [4]-[6], Khrennikov [7, 8], 't Hooft [49] and Anashin [50], [51] 2 The states of the latter are not directly approachable for external inspection. In some models [4, [7, 8, 49] transition from the subquantum (ontic) level to the quantum (observational) level is performed through factorization of the space of subquantum states. Thus the states described by the quantum formalism are treated as the symbolic images of factor classes of ontic states. Moreover, in quantum mechanics there are widely used dynamical systems to model ontic $\mapsto$ epistemic transition, see [4], [52].

This is the good place to remark that recent development of physics is characterized by creation of a number of novel automaton models describing state dynamics. This activity was culminated in 't Hooft's model of totally deterministic dynamical universe [49] functioning as a cellular automaton 3 We also mention

\footnotetext{
${ }^{2}$ At the same time other experts follow the tradition of the Copenhagen school and reject the possibility of emergent treatment of quantum mechanics, see the work of Plotnitsky and Khrennikov for detailed analysis [48].

${ }^{3}$ We emphasize that one has to distinguish sharply theory of cellular automata from general theory of automata used in our paper. The common use of the word "automaton"
} 
the recent work of Anashin [50], [51, an attempt to derive the wave structure of quantum theory from theory of $p$-adic dynamics systems.

Now we tern to molecular biology. The dimension of the space of possible conformation states of proteins is huge and these states are not approachable for direct external inspection. Therefore it is natural to proceed similarly to the onticepistemic approach to quantum mechanics and to work jointly with two coupled models of protein's behavior:

- the ontic model describing dynamics of conformational states;

- the epistemic model describing functional states of a protein.

The dynamics of conformational states is described by an automaton, discrete time and state dynamical machine. Input symbols encode the influences of the environment (the state of the surrounding cell and signaling from other cells). The transition function $f=f(i, s)$ describes transitions between internal states of protein (conformational states) generated by interaction of a protein molecular with the cell-environment; the output function $g=g(i, s)$ describes functional outputs of the protein molecular. The dynamics of functional states is described by the factor-automaton generated through functional equivalence relation on the space of conformational states and the corresponding factorization of the state space and the transition and output functions. Here it is the good place to cite once again Ramanathan et al. 41]: "Motions on the fast time scale allow a protein to sample conformations in the nearby area of its conformational landscape, while motions on slower time scales give it access to conformations in distal areas of the landscape." The mentioned fast scale dynamics is the automaton dynamics of ontic states, slow scale dynamics describes transitions of epistemic states. The later can be slow enough comparing with the fact scale transitions, because any epistemic (functional) state is composed of a large number of ontic states. The protein molecular can spend a lot of time inside such a cluster of conformational states before to jump to the next cluster representing another epistemic state.

This factor-space structure of the space of functional states can lead to nonclassical probabilistic behavior. We remark that in the complete accordance with the ontic-epistemic approach nonclassicality of probability is a purely epistemic feature. At the ontic level, classical probability works well. Such a model can generate very exotic probability distributions, not only quantum-like, cf. [4]-[6], [7, 7], [49], but even so called $p$-adic probability distributions [15]. The latter were invented in $p$-adic (non-Archimedean) physics to provide the probabilistic interpretation of wave functions valued in the fields of $p$-adic numbers or more general

can be misleading. Here we have no possibility to go into details. We just point to the reader that this paper is not about cellular automata! 
non-Archimedean (ultrametric) number fields. Then a few theoretical biological models generating $p$-adic probabilities were invented and theory was completed with numerical simulation. Now we hope that $p$-adic probability theory can find real applications in molecular biology. Of course, these are just preliminary conceptual considerations.

In appendix 2 we presented coupling (due to Anashin) of theory of automata with mappings in the fields of $p$-adic numbers. We tried to make the first steps to describe the class of automata and corresponding $p$-adic maps matching functioning of proteins. This will be the main direction of our further studies. To proceed in this direction, we have to analyze functioning of proteins in more detail, especially from the biological side (and not only dynamical and information processing constraints discussed in appendix 2).

\section{Acknowledgments}

This paper was written under support of the grant of the Faculty of Technology of Linnaeus University, Modeling of Complex Hierarchic systems and the EU-project Quantum Information Access and Retrieval Theory (QUARTZ), Grant No. 721321

\section{Appendix 1: Quantum-like models in biology}

This paper is not about quantum bio-physics, cf. [53, [54]-[56]. It is closer to works on so called quantum-like models exploring the apparatus of quantum probability and information theories to model biological phenomena [57]-[76], [55], [56]. In quantum-like modeling one does not try to study genuine quantum physical processes in a bio-system. The latter is considered as a black box processing information in accordance with the laws of quantum probability and information. Moreover, quantum probabilistic-informational behavior need not be explicitly coupled to quantum physical processes. Quantum-like behavior can be exhibited by macroscopic bio-systems operating at time, space, and temperature scales which do not match the corresponding scales of the quantum physics.

The most successful applications of the quantum-like approach are in modeling brain's functioning, cognition, decision making, psychology, see, e.g., the monographs 62 , 63], 71. Here the brain is treated as a black box with inputs from environment (both physical and mental) and outputs in the form of perceptions, decisions and judgments. The probabilistic-information structure of interrelation between inputs and outputs cannot be described mathematically by the laws of classical probability and information. Therefore alternative ("nonclassical") theories have to be proposed. The quantum formalism is the most well developed, 
advanced and approved in numerous applications. Therefore it is reasonable to apply this formalism as an alternative to the classical probability and information formalisms. This was done in the aforementioned applications outside of physics. Of course, one cannot guarantee that in biological applications nonclassicality coincides with quantumness. It can happen that from the probabilistic and information viewpoints behavior of biological systems is even more complicated than behavior of quantum systems; may be the brain can exhibit informational patterns which structure is more complex than the structure of genuine quantum patterns. May be novel mathematical formalisms have to be designed for biological purposes.

Once again we remind that the quantum-like model of brain's functioning differs from the "quantum physical brain model" in the spitit of R. Penrose [77] and S. Hameroof [78]. The latter tries to connect cognitive actions of the brain at the level of consciousness with physical processes in the brain at the quantum level. The main bio-physical structure involved to this process are micro-tubular. It is claimed that they can be used by the brain for quantum information processing. In particular, they can exhibit such a basic feature of quantum physical systems as entanglement. Justification of this conjecture is the big challenge. For the moment, we cannot reject completely the possibility that the brain really works in this way by exploring quantum physical processes, e.g., at the level of microtubular. However, to justify this theory of brain's functioning one has to solve many problems and some authors presented very heavy arguments that the hot amd macroscopic brain cannot "enjoy" exciting features of the quantum world, see, e.g., Tengmark [79]. However, the creators of the quantum physical brain model argue that quantum features still can be preserved in the brain, at least for timeintervals sufficient to perform a kind of quantum computation leading to cognitive processing of information. The lovely debate have been continued already 30 years. It may take many years to clarify the situation (if such clarification is possible at all in the framework of existing physical theory).

One of the main problems in justification of the quantum-like approach to modeling of cognitive behavior of bio-systems is the resolution of the famous problem of hidden variables. For biologists, we say a few words about this problem.

The state $\psi$ of a classical physical system determines the outcomes of all observables which can be measured for this state. Observables are represented as functions on the state space, $\psi \rightarrow f(\psi)$. For example, in classical mechanics the state $\psi$ is given by the pair $\psi=(x, p)$, position and momentum (the coordinates in phase space). Here observables can be treated as functions on the phase space, $f=f(x, p)$. In classical statistical mechanics the state $\rho$ is represented by the probability distribution on the phase space; observables are also defined as functions on the phase space. This kind of states, statistical states, do not determine the measurement outcomes, but only the probabilities of these outcomes. Thus the 
average of the observable represented by the function $f$ in the state given by the probability distribution $\rho$ is calculated by the rule of classical probability theory:

$$
\langle f\rangle_{\rho}=\int f(x, p) d \rho(x, p) .
$$

If the probability has the density $\rho(x, p)$, then the average is given by the integral: $\langle f\rangle_{\rho}=\int f(x, p) \rho(x, p) d x d p$.

The state of a quantum system is represented by a (normalized) vector $\psi$ of complex Hilbert space $H$, i.e., $\langle\psi \mid \psi\rangle=1$, where $\left\langle\phi_{1} \mid \phi_{2}\right\rangle$ denotes the scalar product of vectors $\phi_{1}, \phi_{2} \in H$. Quantum observables are represented by Hermitian operators. Similarly to the state used in classical statistical mechanics, it determines only the probabilities of outcomes. However, in contrast to classical statistical mechanics, the quantum average is represented as

$$
\langle A\rangle_{\psi}=\langle A \psi \mid \psi\rangle \text {. }
$$

From the very beginning of quantum mechanics, the following question disturbed its fathers: Is it possible to introduce a quantum analog of the classical phase space? More generally this is the question about the possibility of functional representation of quantum observables and measure-theoretic representation of quantum states. In particular, one wonders whether it is possible to represent quantum average (9) in the classical integral form (86).

Since for the moment physical parameters determining the outputs of measurements are unknown 4, they got the name hidden variables. Two orthogonal positions were presented by Einstein and Bohr, respectively. Einstein was sure that such variables determining the values of quantum observables exist and this is just a matter of time, soon or latter the formalism with hidden variables reproducing quantum measurement theory will be designed. Bohr was sure that this will never happen, hidden variables do not exist. Bohr's position was merely philosophical. Later it was transformed into mathematical statements known as "no-go" theorems. The first of them was proven by J. von Neumann. However, it was criticized as unphysical. The most famous no-go theorem was formulated by J. Bell. It is based on the inequality for correlations known as Bell's inequality. A theory with hidden variables should produce correlations which satisfy this inequality. But the quantum formalism implies correlations violating this inequality. The experiment seems to be in favor of the quantum prediction. We emphasize that Bell's no-go

\footnotetext{
${ }^{4}$ In contrast to classical mechanics, the pair position-momentum, $\psi=(x, p)$, cannot serve as such parameters, because the position and momentum cannot be measurement jointly. By the orthodox version of the Copenhagen interpretation they even "do not exist".
} 
theorem differs crucially from von Neumann's theorem. Bell lifted up the issue of nonlocality which first mentioned by Einstein. This issue was not present in von Neumann's theorem. The main point in our discussion is that a nonlocal hidden variable model can match the predictions of quantum mechanics. Correlations based on signaling between subsystems can violate Bell's inequality. In physics such signaling is impossible, because subsystems, e.g., two entangled photons, are separated for a long distance such that signaling should have the speed extending the speed of light. Therefore quantum nonlocality appears as a mystical physical phenomenon, the spooky action at a distance.

Now we turn again to biology. In contrast to quantum physics, here "hidden variables do exist". Moreover, they are not hidden at all. These are states of neurons in brain science, states of proteins, genes, cells in molecular biology and so on. It seems that those who apply the quantum-like approach are in trouble. Quantum theory says that there are no (local) hidden variables, but biology says that they exist (and not even hidden). The key word here is (non)local. In biology we need not be afraid of nonlocal models, because they are nonlocal only formally. In reality nonlocal contributions to observations can be generated by signaling between subsystems, e.g., between cells. This signaling is not mystical, since its speed does not exceeds the speed of light. The crucial point is that biological systems are negligibly small in size (comparing with light's velocity), cf. [66]. Thus we can apply the quantum formalism to biological phenomena without to collide with no-go theorems!

As was pointed out, quantum-like modeling was very successful i modeling of cognition and decision making. However, recently quantum operational methods were extended to model behavior of micro-biological systems, e.g., cells, genomes, epigenomes, and proteins [80, 81]. Thus we can speak about quantum information biology as unifying information processing at all scales, from molecular biology to cognition and social science, see [82], 83] for details.

\section{Appendix 2: $P$-adic mapping representation of epistemic automata}

In this section following V. Anashin [2] we shall consider representation of apparata by mappings of the ring of $p$-adic numbers $\mathbf{Z}_{p}$. We start with presenting theory of automata in more detail. We remind that the initial automaton $\mathfrak{A}\left(s_{0}\right)=\left\langle I, S, V, f, g, s_{0}\right\rangle$ is an automaton, where one state $s_{0} \in S$ is fixed; $s_{0}$ is called the initial state. In particular, the Mealy machines [46] (and hence the Moore machines [47) are initial automata with finite state spaces. 


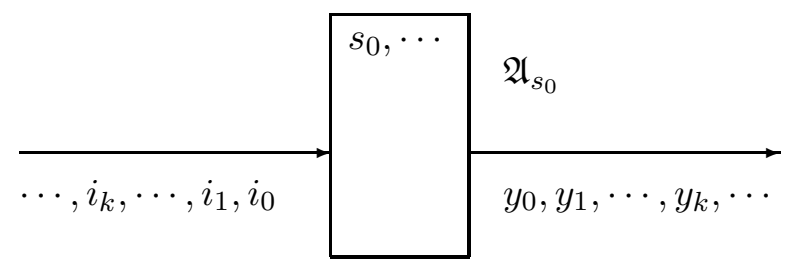

Figure 1: Functioning of initial automation

In theory of automata it is often assumed that there exists a state $s_{0} \in S$ such that all the states of the automaton $\mathfrak{A}$ are reachable from $s_{0}$; that is, given state $s \in S$, there exists input word $w$ over alphabet $I$ such that after the word $w$ has been fed to the automaton $\mathfrak{A}\left(s_{0}\right)$, the automaton reaches the state $s$.

To the automaton $\mathfrak{A}$ we put into a correspondence the family of all initial automata $\mathfrak{A}(s)=\langle I, S, V, f, g, s\rangle, s \in S$.

Now we assume that both alphabets $I$ and $O$ are $p$-element alphabets: $I=$ $O=\{0,1, \ldots, p-1\}=F_{p}$; so further the word automaton stands for initial automaton with input/output alphabets $F_{p}$. A typical example of an automaton of that sort is the 2-adic adding machine, $\mathfrak{A}(1)=\left\langle F_{2}, F_{2}, F_{2}, f, g, 1\right\rangle$, where $f(i, s)=i s(\bmod 2), \mathrm{g}(\mathrm{i}, \mathrm{s})=\mathrm{i}+\mathrm{s}(\bmod 2)$ for $s \in S=F_{2}, i \in I=F_{2}$.

Given an automaton $\mathfrak{A}(s)=\langle I, S, V, f, g, s\rangle$, the automaton transforms input words (with respect to the alphabet $F_{p}$ ) of length $n$ into output words of length $n$; that is, $\mathfrak{A}(s)$ maps the set $W_{n}$ of all words of length $n$ into $W_{n}$; we denote corresponding mapping via $\mathfrak{F}_{\mathfrak{A} ; n}(s)$. It is clear now that behaviour of the automaton can be described in terms of the mappings $\mathfrak{F}_{\mathfrak{A} ; n}(s)$ for all $s \in S$ and all $n \in \mathbf{N}=$ $\{1,2,3, \ldots\}$. If all states of the automaton are reachable from the state $s_{0}$, it suffices to study only the mappings $\mathfrak{F}_{\mathfrak{A} ; n}\left(s_{0}\right)$ for all $n \in \mathbf{N}$.

Of course, here "suffices" has the epistemic meaning, i.e., by considering the mappings $\mathfrak{F}_{\mathfrak{A} ; n}\left(s_{0}\right)$, instead of the original automaton, we neglect by the dynamics of the ontic states; in our case the dynamics of conformational states. We also make the following methodological comment. The ideology of representation of an automaton by mappings $\mathfrak{F}_{\mathfrak{A} ; n}\left(s_{0}\right)$ is different from the ideology presented in this paper: transition from the ontic automaton to the epistemic one through factorization of the ontic state space. By moving to the $\mathfrak{F}_{\mathfrak{A} ; n}\left(s_{0}\right)$-functional representation we neglect the state dynamics.

The system of functions $\mathfrak{F}_{\mathfrak{A} ; n}\left(s_{0}\right)$ can be considered as the mapping in the space of infinite words in the alphabet $F_{p}$. The latter can be identified with the ring of $p$-adic integers $\mathbf{Z}_{p}$. Thus each automaton can be represented by a mapping $u=$ $\mathfrak{F}_{\mathfrak{A}}: \mathbf{Z}_{p} \rightarrow \mathbf{Z}_{p}, u(x)=\sum_{k=0}^{n} \delta_{k}(x) p^{k}$, where $x=\sum_{k=0}^{n} x_{k} p^{k}$ and $\delta_{k}, k=0,1,2 \ldots$, are "coordinate functions" valued in $F_{p}$.

Mappings generated by automata have very special structure. This structure 
is encoded in the coordinate functions. Here $\delta_{k}$ depends only on the coordinates $x_{0}, \ldots, x_{k}$ of the variable $x: \delta_{k}=\delta_{k}\left(x_{0}, \ldots, x_{k}\right)$. In the protein model this means that protein cannot feel future states of the environment; its state at the instance of time $t_{k}=k$ depends only on environment's states at the previous instances of time $t=0,1, \ldots, k$. It can be easily shown that such function $u=\mathfrak{F}_{\mathfrak{A}}: \mathbf{Z}_{p} \rightarrow \mathbf{Z}_{p}$ satisfies 1-Lipschitz condition:

$$
\rho_{p}(u(x), u(y)) \leq \rho_{p}(x, y), x, y \in \mathbf{Z}_{p},
$$

where $\rho_{p}$ is the $p$-adic distance. This class of functions in relation to theory of automata was introduced by V. Anashin [13, [16, [18, [2], [3] and it plays the important role in theory of $p$-adic dynamical systems, e.g., [84, 85]. We call the space of 1-Lipschitz functions the Anashin functional space. Thus in the epistemological model one can in principle consider just a mapping $\mathfrak{F}_{\mathfrak{A}}$ belonging to the Anashin space (if we are not interested in the dynamics of epistemic states). The following natural question arises:

Can any mapping belonging to Anashin's space be generated by some automaton?

The answer is 'yes', but in general the state space of the automaton can be infinite [2]. As we have already discussed, by treating protein-automata as computational machines we have to restrict modeling to finite state spaces. At the same time physics gives us arguments in favor of infinite and even continuous state spaces.

The problem of description of functions representing finite automata in terms of generated $p$-adic mappings were solved by Vuillemin [20] (for $p=2$ ), Smyshlyaeva [21] and Anashin [3] (for an arbitrary $p$ ). Their solutions, although beautiful mathematically (and based on the use of coordinate functions and van der Put series, respectively), are too technical and too abstractly formulate to be applicable to our biological modeling.

Now we discuss other special features of automata modeling of proteins' behavior (in Anashin-like manner, i.e., by using the representation by $p$-adic functions). We have already pointed out that the Moore machines seems to be more natural for such modeling than the general Mealy machines. However, in the $p$-adic functional approach the difference between these two types of finite states machines disappears: the classes of functions representing these machines coincide. For any function representing a Mealy machine, it is possible to construct a Moore machine representable by this function (of course, the inverse statement is also true). This feature of Anashin's representation is not so natural for our approach to proteins' modeling. However, the real situation is more complicated, since we are not sure that the space of protein's conformational states can be represented adequately as a finite state space. Thus it may be that we have to proceed with "generalized 
Moore machines" having infinite state spaces. We do not know whether functional classes representing such machines and general automata coincide or not.

The aforementioned result about the coincidence of $p$-adic functional classes for the Moore and Mealy machines shows that the functional approach, although very powerful from the mathematical viewpoint, shadows the internal structure of an automaton. We would like to mention another problem which is especially important for non-finite machines. The state space of a biological or physical system is not only a set of point; typically it is endowed with some topological structure which plays the important role in understanding of functioning of this system. The simplest examples are state spaces $S=\left\{s_{1}, \ldots, s_{n}, \ldots\right\}$ with the discrete topology and $S=\mathbf{R}$ with its continuous topology. It seems that this difference in topological structures of state spaces disappear in the functional representation. However, may be a deeper analysis can clarify this connection between topology on $S$ and the special features of the corresponding $p$-adic mapping $\mathfrak{F}_{\mathfrak{A}}$. For example, suppose that $S=\mathbf{Z}_{p}$ and that $f: \mathbf{Z}_{p} \times \mathbf{Z}_{p} \rightarrow \mathbf{Z}_{p}$ and $g: \mathbf{Z}_{p} \times \mathbf{Z}_{p} \rightarrow \mathbf{Z}_{p}$ are continuous functions. May be the corresponding mapping $\mathfrak{F}_{\mathfrak{A}}: \mathbf{Z}_{p} \rightarrow \mathbf{Z}_{p}$ has some special features? May be the difference can be monitored not at the level of a topological structure, but a differential structure on $S$ ? For example, $\mathfrak{F}_{\mathfrak{A}}: \mathbf{Z}_{p} \rightarrow \mathbf{Z}_{p}$ is differentiable if both $f$ and $g$ are differentiable.

We continue the discussion on determination of the subspace of the Anashin functional space corresponding to "protein automata." In general theory of automata one of the commonly used classes is the class of transitive automata. We recall that a family $U$ of mappings of a finite non-empty set $M$ into $M$ is called transitive whenever given a pair $(a, b) \in M \times M$, there exists $u \in F$ such that $u(a)=b$. An automaton is transitive if it can generate an arbitrary output from an arbitrary input. Anashin [2] characterized transitive automata in terms of ergodicity of corresponding mappings from $\mathbf{Z}_{p}$ to $\mathbf{Z}_{p}$. Since for our protein-modeling we are interested in finite-automata, we make two remarks on interrelation of ergodicity and the possibility of the finite-automata realization [2], 3]:

- There exist ergodic mappings belonging to the Anashin class which are not representable by finite automata. For example, ergodic polynomials of degrees $>1$ cannot be represented by finite automata.

- An ergodic polynomial of the degree one, i.e., an affine mapping of the form $f(x)=a x+b$ can be represented by a finite automaton if and only if its coefficients $a, b$ are rational $p$-adic numbers, i.e., they belong to the intersection of $\mathbf{Z}_{p}$ and $\mathbf{Q}$, where $\mathbf{Q}$ is the field of rational numbers 5

${ }^{5}$ The number $x$ belonging to $\mathbf{Z}_{p}$ is rational if and only if its representation by the sequence of digits, see (5), is periodic, i.e., some block of digits is repeated periodically. 
We remark that consideration of mappings determined by rational parameters matches with the ideology of $p$-adic theoretical physics, see, for example, [86][89], [15]. Here only rational numbers are treated as "physical numbers", numbers which can get experimental verification.

However, this very useful class of automata, transitive-automata, does not match with our protein model. As was discussed at the very end of section 8 , a protein is very specialized machine and its reacts very concretely to any input pattern of the environment producing very special functional output. Thus proteins have to be modeled by non-transitive automata.

The simplest non-transitive automata are given by locally constant mappings, i.e., continuous mappings taking only finitely many values. We remark that in the $p$-adic and more generally ultrametric case the characteristic function of a ball is continuous! A locally constant mapping can be represented as a linear combination of such characteristic functions, i.e., $\mathbf{Z}_{p}$ can be represented as the disjoint union of (finitely many) balls such that this mapping takes the constant value at each ball. Locally constant functions play the important role in the $p$-adic (ultrametric) analysis. They form the dense subspace in the space of all continuous functions.

In section 9.2 starting with the ultrametric structure of the ontic state space we proposed representation of the epistemic state space as the space of balls. Therefore it is natural to assume that the output function (observational function) $g$ is locally constant on the ontic state space $S$. Therefore it is natural as well to suppose that the realization of the protein-automaton by a mapping $\mathfrak{F}_{\mathfrak{A}}: \mathbf{Z}_{p} \rightarrow \mathbf{Z}_{p}$ is also a locally constant function. Now the following question arises: Which locally constant functions can be realized by finite automata? The answer is known [2]: only functions taking the $p$-adic rational values. This again matches with the ideology of the $p$-adic theoretical physics.

Under the assumption that behavior of proteins can be mathematically modeled with the aid of the Mealy machines (finite state automata), we can guess that the class of protein-automata is a subclass of the class of automata for which $\mathfrak{F}_{\mathfrak{A}}$ is a locally constant $p$-adic rational-valued function. However, to describe this subclass precisely we have to analyze this problem taking into account more delicate biological and molecular features of representation of proteins by automata. In general characterization of the class of automata matching proteins' behavior is the interesting mathematical problem. And we hope that publication of this concept-paper would attract interest of mathematicians and molecular biologists to study of this problem.

This characterization is similar to characterization of rational numbers in the field of real numbers $\mathbf{R}$ : these are numbers having periodic decimal representations. 


\section{References}

[1] Sakarovitch, J. 2009. Elements of Automata Theory. Cambridge University Press, Cambridge.

[2] Anashin, V., 2012. Non-Archimedean theory of discrete systems. Math. Computer Sc. 6, 375-393.

[3] Anashin, V., 2012. Automata finiteness criterion in terms of van der Put series of automata functions. P-Adic Numbers, Ultrametric Analysis Appl. 4, 151-160.

[4] Atmanspacher, H., Bishop, R. C., and Amann, A., 2001. Extrinsic and intrinsic irreversibility in probabilistic dynamical laws. In: Khrennikov, A. (Ed.), Foundations of Probability and Physics. WSP, Singapore, pp. 50-70.

[5] Atmanspacher, H., 2002. Determinism is ontic, determinability is epistemic. In: Atmanspacher, H. and Bishop, R. C. (Eds.), Between Chance and Choice: Interdisciplinary Perspectives on Determinism. Imprint Academic, Thorverton UK, pp. 49-74.

[6] Atmanspacher, H. and Primas, H., 2005. Epistemic and ontic quantum realities. In: Khrennikov, A. (Ed.), Foundations of Probability and Physics-3. AIP, Melville, NY, 750, pp. 49-62

[7] Khrennikov, A., 2003. Contextual viewpoint to quantum stochastics. Math. Phys. 44, 2471- 2478.

[8] Khrennikov, A., 2003. Representation of the Kolmogorov model having all distinguishing features of quantum probabilistic model. Phys. Lett. A 316, 279-296.

[9] Khrennikov, A., 2004. Contextual approach to quantum mechanics and the theory of the fundamental prespace. J. Math. Phys. 45, 902-921.

[10] Fischer, E. 1894. Einfluss der Configuration auf die Wirkung den Enzyme. Ber. Deutsch. Chem. Ges. 27, 2985-2993.

[11] Redfern, O. C., Dessailly, B., Orengo, C. A., 2008. Exploring the structure and function paradigm. Curr. Opin. Struct. Biol. 18(3), 394-402.

[12] Dunker, A. K., Lawson, J. D., Brown, C. J., Williams, R. M., Romero, P., Oh, J. S., Oldfield, C. J., Campen, A. M., Ratliff, C. M., Hipps, K. W., Ausio, J., Nissen, M. S., Reeves, R., Kang, C., Kissinger, C. R., Bailey, R. W., Griswold, 
M. D., Chiu, W., Garner, E. C., Obradovic, Z., 2001. Intrinsically disordered protein. J. Molecular Graphics and Modelling 19 (1), 26-59.

[13] Anashin, V., 1994. Uniformly distributed sequences of $p$-adic integers. Math. Notes 55, 109-133.

[14] De Smedt, S., Khrennikov, A., 1997. Dynamical systems and theory of numbers. Comment. Math. Univ. St. Pauli 4(6), 117-132.

[15] Khrennikov, A., 1997. Non-Archimedean Analysis: Quantum Paradoxes, Dynamical Systems and Biological Models. Kluwer, Dordreht.

[16] Anashin, V., 2002. Uniformly distributed sequences of $p$-adic integers, II. Discrete Math. Appl. 12(6), 527-590.

[17] Khrennikov, A.Yu. and Nilson, M., 2004. P-adic Deterministic and Random Dynamics. Springer, Dordrecht.

[18] Anashin, V., 2006. Ergodic Transformations in the Space of $p$-adic Integers. AIP Conf. Proc., 826, 3-24.

[19] Fan, A.-H., Liao, L., Wang, Y. F., Zhou, D., 2007. $P$-adic repellers in $Q_{p}$ are subshifts of finite type. C. R. Math. Acad. Sci. Paris 344, 219-224.

[20] Vuillemin, J., 2000. Finite digital synchronous circuits are characterized by 2-algebraic truth tables. Lect. Notes Comp. Sc. 1961, 1-7.

[21] Smyshlyaeva, T. I., 2013. A criterion for functions defined by automata to be bounded-determinate, Diskret. Matem. 25, 121-134.

[22] Kozyrev, S. V., 2006. Ultrametric dynamics as a model of interbasin kinetics. J. Contemp. Math. Anal. 41, 38-48.

[23] Kozyrev, S. V., 2006. Ultrametric analysis and interbasin kinetics. AIP Conf. Proc., Melville, NY, 826, 121-128.

[24] Kozyrev, S. V., 2010. Dynamics on rugged landscapes of energy and ultrametric diffusion. P-Adic Numbers, Ultrametric Analysis Appl. 2, 122-132.

[25] Dragovich, B. and Dragovich, A., 2009. A p-Adic model of DNA sequence and genetic code. P-Adic Numbers, Ultrametric Analysis Appl. 1, 34-41.

[26] Khrennikov, A., 2009. Gene expression from polynomial dynamics in the 2adic information space. Chaos, Solitons, and Fractals 42, 341-347. 
[27] Dragovich, B. and Dragovich, A., 2010. P-adic modelling of the genome and the genetic code. Computer J. 53, 432-442.

[28] Khrennikov, A. Yu., 1998. Human subconscious as the p-adic dynamical system. J. Theor. Biol. 193, 179-196.

[29] Albeverio, S., Khrennikov, A., and Kloeden, P. E., 1999. Memory retrieval as a p-adic dynamical system. BioSystems 49. 105-115.

[30] Khrennikov, A.Yu., 2010. Modelling of psychological behavior on the basis of ultrametric mental space: Encoding of categories by balls. $P$-adic Numbers, Ultrametric Analysis Appl. 2, 1-20.

[31] Khrennikov, A.Yu., 2014. Cognitive processes of the brain: An ultrametric model of information dynamics in unconsciousness. P-adic Numbers, Ultrametric Analysis Appl. 6, 293-302.

[32] Murtagh, F., 2012. Ultrametric model of mind, I: Review. P-adic Numbers, Ultrametric Analysis and Appl. 4, 193-206.

[33] Murtagh, F., 2012. Ultrametric model of mind, II: Application to text content analysis. P-adic Numbers, Ultrametric Analysis Appl. 4, 207-221.

[34] Murtagh, F., 2013. The new science of complex systems through ultrametric analysis: Application to search and discovery, to narrative and to thinking. $P$-adic Numbers, Ultrametric Analysis Appl. 5 (4), 326-337.

[35] Iurato, G., 2013. Mathematical thought in the light of Matte Blanco work. Phil. Math. Ed. J. 27, 1-9.

[36] Iurato, G., 2015. A brief comparison of the unconscious as seen by Jung and Levy-Strauss. Anthropology Cons. 26, 60-107.

[37] Khrennikov, A., 2016. Probability and Randomness: Quantum versus Classical. WSP, Singapore.

[38] Khrennikov, A. Yu., 1994. An algorithmic approach to $p$-adic probability theory. Dokl. Akad. Nauk 335, 35-38.

[39] Dyson, H. J. and Wright, P. E., 2005. Intrinsically unstructured proteins and their functions. Nat. Rev. Mol. Cell Biol. 6 (3), 197-208.

[40] Dunker, A. K., Silman, I., Uversky, V. N., Sussman, J. L., 2008. Function and structure of inherently disordered proteins. Curr. Opin. Struct. Biol. 18 (6), 756-764. 
[41] Ramanathan, A., Savol, A., Burger, V., Chennubhot, C. S. and Agarwal, P. K., 2014. Protein conformational populations and functionally relevant substates, Acc. Chem. Res., 47 (1), 149-156.

[42] Henzler-Wildman, K. A., Lei, M., Thai, V., Kerns, S. J., Karplus, M., Kern, D. A., 2007. Hierarchy of Timescales in Protein Dynamics Is Linked to Enzyme Catalysis. Nature 450, 913-916.

[43] Hammes, G. G., Benkovic, S. J., Hammes-Schiffer, S., 2011. Flexibility, diversity, and cooperativity: Pillars of enzyme catalysis. Biochemistry 50, 1042210430.

[44] Ramanathan, A., Agarwal, P. K., 2011. Evolutionarily conserved linkage between enzyme fold, Flexibility, and Catalysis. PLoS Biol. 9, No. e1001193.

[45] Ramanathan, A., Savol, A. J., Langmead, C. J., Agarwal, P. K., Chennubhotla, C. S., 2011. Discovering conformational sub-states relevant to protein function. PLoS ONE 6, No. e15827.

[46] Mealy, G. H., 1955. A method for synthesizing sequential circuits. Bell Syst. Tech. J. 34, 1045-1079.

[47] Moore, E. F., 1956. Gedanken-experiments on sequential machines. Automata Stud., Annals of Math. Stud. 34, 129-153.

[48] Plotnitsky, A. and Khrennikov, A., 2015. Reality without realism: On the ontological and epistemological architecture of quantum mechanics. Found. Phys. 45, 1269-1300.

[49] 't Hooft, G., 2016. The Cellular Automaton Interpretation of Quantum Mechanics. Springer, Berlin-Heidelberg-New York.

[50] Anashin, V., 2015. Quantization causes waves: Smooth finitely computable functions are affine. P-adic Numbers, Ultrametric Analysis Appl. 7, 169-227.

[51] Anashin, V.S., 2015. Smooth finitely computable functions are affine, or why quantum systems cause waves, Doklady Math. 92, 655-657.

[52] Khrennikov, A., 2005. Schrödinger dynamics as the Hilbert space projection of a realistic contextual probabilistic dynamics. Europhys. Lett. 69(5), 678-684.

[53] Arndt, M., Juffmann, Th, Vedral, V., 2009. Quantum physics meets biology. HFSP J. 3 (6), 386e400. 
[54] Melkikh, A.V., Seleznev, V.D., 2012. Mechanisms and models of the active transport of ions and the transformation of energy in intracellular compartments. Prog. Bioph. Mol. Biol. 109, 33-57.

[55] Melkikh, A.V., Khrennikov, A., 2015. Nontrivial quantum and quantum-like effects in biosystems: unsolved questions and paradoxes. Progress in Biophysics and Molecular Biology. 119. issue 2, 137-161.

[56] Melkikh, A.V., Khrennikov, A., 2016. Quantum-like model of partially directed evolution. Progress in Biophysics and Molecular Biology. 10.1016/j.pbiomolbio.2016.12.005.

[57] Khrennikov, A., 2004. On quantum-like probabilistic structure of mental information. Open Systems and Information Dynamics 11(3), 267-275.

[58] Asano, M., Ohya, M. and Khrennikov, A., 2010. Quantum-like model for decision making process in two players game. Found. Phys. 41, 538-548.

[59] Asano, M., Ohya, M., Tanaka, Y., Khrennikov, A. and Basieva, I., 2011. Dynamics of entropy in quantum-like model of decision making, J. Theor. Biol. 281, 56-64.

[60] Asano, M., Basieva, I., Khrennikov, A., Ohya, M., Tanaka, Y., 2012. Quantum-like generalization of the Bayesian updating scheme for objective and subjective mental uncertainties. J. Math. Psych. 56, 166-175.

[61] Khrennikov, A., 2006. Quantum-like brain: Interference of minds. BioSystems $84,225-241$.

[62] Khrennikov, A., 2010. Ubiquitous Quantum Structure: from Psychology to Finance. Springer, Heidelberg-Berlin-New York.

[63] Busemeyer, J. R. and Bruza, P. D., 2012. Quantum Models of Cognition and Decision. Cambridge, Cambridge Press.

[64] Busemeyer, J. B., Wang, Z. and Townsend, J. T., 2006. Quantum dynamics of human decision making. J. Math. Psych. 50, 220-241.

[65] Dzhafarov, E.N. and Kujala, J.V., 2012. Selectivity in probabilistic causality: Where psychology runs into quantum physics. J. Math. Psych. 56, 54-63.

[66] de Barros, A., J. and Suppes, P., 2009. Quantum mechanics, interference, and the brain. J. Math. Psych. 53, 306-313. 
[67] de Barros, A. J., 2012. Joint probabilities and quantum cognition. In: Khrennikov, A., Atmanspacher, H., Migdall, A. and Polyakov, S. (Eds.), Quantum Theory: Reconsiderations of Foundations-6. AIP, Melville, NY, 1508, pp. 98104.

[68] Atmanspacher, H., Filk, Th., 2012. Temporal nonlocality in bistable perception. In: Khrennikov, A., Atmanspacher, H., Migdall, A. and Polyakov, S. (Eds.), Quantum Theory: Reconsiderations of Foundations-6. AIP, Melville, NY, 1508, pp. 79-88.

[69] Busemeyer, J. R., Pothos, E. M., Franco, R. and Trueblood, J., 2011. A quantum theoretical explanation for probability judgment errors. Psych. Rev. 118, 193-218.

[70] Cheon, T. and Takahashi, T., 2010. Interference and inequality in quantum decision theory. Phys. Lett. A 375, 100-104.

[71] Haven, E. and Khrennikov, A., 2012. Quantum Social Science. Cambridge Press, Cambridge.

[72] Khrennikova, P., Haven, E. and Khrennikov, A., 2014. An Application of the Theory of Open Quantum Systems to Model the Dynamics of Party Governance in the US Political System. Int. J. Theor. Phys. 53. 1346-1360.

[73] Khrennikova, P., 2012. Evolution of quantum-like modeling in decision making processes. AIP Conf. Proc. 1508, 108-112.

[74] Khrennikova, P. and Haven, E., 2016. Instability of political preferences and the role of mass media: a dynamical representation in a quantum framework. Phil. Tr. Royal Soc. A 374, 20150106.

[75] Khrennikova, P., 2016. Quantum dynamical modeling of competition and cooperation between political parties: The coalition and non-coalition equilibrium model. J. Math. Psych. 71, 39-50 .

[76] Khrennikova, P., 2016. Application of quantum master equation for long-term prognosis of asset-prices. Physica A: Stat. Mech. Appl. 450, 253-263 .

[77] Penrose, R., 1989. The Emperor's New Mind. Oxford Univ. Press, New-York.

[78] Hameroff, S., 1994. Quantum coherence in microtubules. A neural basis for emergent consciousness? J. of Cons. Stud. 1, 91-118.

[79] Tegmark, M., 2000. Importance of quantum decoherence in brain processes. Phys. Rev. E 61(4), 4194-4206. 
[80] Basieva, I., Khrennikov, A., Ohya, M. and Yamato, I., 2011. Quantum-like interference effect in gene expression glucose-lactose destructive interference. Syst. Synth. Biol. 5, 5968.

[81] Asano, M., Basieva, I., Khrennikov, A., Ohya, M., Tanaka, Y. and Yamato, I., 2012. Quantum-like model for the adaptive dynamics of the genetic regulation of E-coli's metabolism of glucose/lactose. Syst. Synth. Biol. 6, 1-7.

[82] Asano, M., Khrennikov, A., Ohya, M., Tanaka, Y. and Yamato, I., 2015. Quantum Adaptivity in Biology: from Genetics to Cognition. Springer, Heidelberg-Berlin-New York.

[83] Asano, M., Basieva, I., Khrennikov, A., Ohya, M., Yamato, I., 2013. NonKolmogorovian approach to the context-dependent systems breaking the classical probability law. Found. Phys. 43, 895-911.

bibitemvander1 Khrennikov, A. and Yurova, E., 2013. Criteria of measurepreserving for p-adic dynamical systems in terms of the van der Put basis. J. Number Theor. 133, 484-491.

[84] Yurova, E.I., 2012. On measure-preserving functions over $\mathbf{Z}_{3}$. P-adic Numbers, Ultrametric Analysis Appl. 4, 326-335.

[85] Yurova, E. I., 2010. Van der Put basis and $p$-adic dynamics, $P$-adic Numbers, Ultrametric Analysis Appl. 2 (2), 175-178.

[86] Vladimirov, V. S., Volovich, I.V. and Zelenov, E.I., 1994. P-adic Analyisis and Mathematical Physics, (World Scientific, Singapore).

[87] Khrennikov, A., 1994. P-adic Valued Distributions and Their Applications to the Mathematical Physics. Kluwer, Dordreht.

[88] Dragovich, B., 1995. Adelic harmonic oscillator. Int. J. Mod. Phys. A 10, 2349-2365.

[89] Albeverio, S., Cianci, R., Khrennikov, A.Yu., 2009. p-adic valued quantization. P-adic Numbers, Ultrametric Analysis Appl. 1, 91-104. 